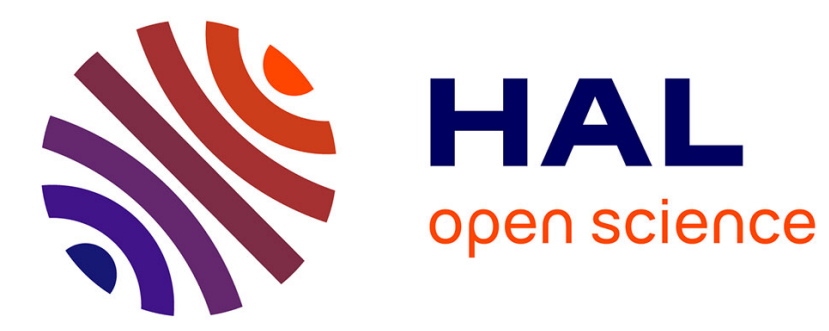

\title{
Savoirs théoriques et production de discours dans les écoles supérieures d'art
}

\author{
J. Vandenbunder
}

\section{To cite this version:}

J. Vandenbunder. Savoirs théoriques et production de discours dans les écoles supérieures d'art. Marges - Revue d'art contemporain, 2016, L'Artiste-théoricien, 22, pp.87-98. 10.4000/marges.1112 . hal-01462903

\section{HAL Id: hal-01462903 https://hal.science/hal-01462903}

Submitted on 15 Feb 2017

HAL is a multi-disciplinary open access archive for the deposit and dissemination of scientific research documents, whether they are published or not. The documents may come from teaching and research institutions in France or abroad, or from public or private research centers.
L'archive ouverte pluridisciplinaire HAL, est destinée au dépôt et à la diffusion de documents scientifiques de niveau recherche, publiés ou non, émanant des établissements d'enseignement et de recherche français ou étrangers, des laboratoires publics ou privés. 
Titre : Savoirs théoriques et production de discours dans les écoles supérieures d'art

Auteur : Jérémie Vandenbunder

\title{
Résumé :
}

Cet article considère le statut des enseignements théoriques dans les écoles d'art et la façon dont ceux-ci interviennent dans les mécanismes de la socialisation professionnelle. Il met en lumière la façon dont les étudiants en art découvrent les représentations du groupe professionnel et comment celles-ci introduisent un rapport particulier avec le discours théorique. Il introduit également la question du processus de Bologne et les débats que celui-ci a pu susciter dans les écoles d'art.

\begin{abstract}
:
This article considers the status of theoretical teaching in art schools and how they are involved in the mechanisms of professional socialization. It highlights how art students discover the representations of the professional group and how these introduce a special relationship with the theoretical discourse. It also introduces the issue of the Bologna process and the debates that it may have caused in the art schools.
\end{abstract}

\section{Notice biographique :}

Jérémie Vandenbunder est docteur en sociologie. Il est membre du laboratoire Printemps à l'université de Versailles - Saint Quentin en Yvelines. Il a publié en 2011 un article intitulé «Une école de la maturation ? Analyse de la formation artistique entre immédiateté du don, rythme des études et processus de création »dans la revue Temporalités et il a contribué en 2014 à l'ouvrage Faire l'art. Analyser les processus de créations artistiques publié chez L'Harmattan.

\section{Article :}

Introduction

Dans la seconde moitié du XVIIe siècle, lorsque l'école de l'Académie de peinture et de sculpture fut créée, celle-ci avait pour but premier de concurrencer la maitrise, corporation d'artisans qui réglementait alors l'accès aux professions de peintre et de sculpteur. C'est que l'enseignement des arts se faisaient alors principalement dans des ateliers privés, sous l'autorité (et au profit) du maitre. Dès lors, en ouvrant une école, l'Académie pouvait apporter une forme différente d'enseignement, s'éloignant des techniques pour s'ouvrir sur une dimension plus intellectuelle. Ainsi, France Nerlich 
souligne à propos de l'Académie et de son enseignement qu'il s'agissait alors de « développer une approche intellectuelle plus affirmée des beaux-arts » et «d'offrir un accès à autre chose qu'à la routine d'atelier, de transmettre une idée de l'art plus que le métier lui-même. ${ }^{1}$ Cinq siècles plus tard, cette division est toujours opérante. Certes, les corporations d'artisans n'existent plus mais il reste que les écoles supérieures d'art, héritières de l'enseignement académique, dispensent encore aujourd'hui un enseignement qui se veut tout autant théorique que technique. Elles se distinguent ainsi des ateliers d'amateurs ou des écoles municipales, exclusivement centrées sur la technique. Comme nous allons tenter de le démontrer dans cet article, cela a de nombreuses implications pour les enseignants, les étudiants et les écoles d'art elles-mêmes.

Il existe aujourd'hui une quarantaine d'écoles supérieures d'art en France, sous tutelle du ministère de la Culture et de la Communication. Elles accueillaient en 2012-2013 près de 10000 étudiants, pour des cursus de trois et cinq ans. Si les curriculums des écoles d'art accordent une large part aux différentes techniques plastiques, notamment en première année, les enseignements théoriques qu'elles délivrent sont loin d'être anecdotiques. Allant de l'histoire de l'art aux langues étrangères, de l'esthétique à la sémiotique, l'ensemble des disciplines enseignées, par des professeurs le plus souvent issus du monde universitaire, est particulièrement large. Aujourd'hui, cette place de l'enseignement théorique est au centre des préoccupations dans les écoles d'art du fait de l'introduction depuis 2009 de la réforme LMD dans ces établissements. Les différentes conséquences de cette réforme, et notamment la question des formes de la recherche menée dans les écoles, ont conduit à de nombreux débats et interrogations dans le monde de l'enseignement artistique et même au-delà. C'est que ceci nous amène de fait à considérer quelle place la théorie occupe dans l'enseignement artistique et dans l'art en général et, plus particulièrement, de quelle manière cette théorie est assimilable à celle qui est enseignée dans le monde universitaire.

Pour ce qui concerne la présente étude, elle se concentrera principalement sur les différentes implications que cette question de la théorie peut avoir sur l'expérience et les représentations des étudiants en école d'art. En nous appuyant sur un travail de thèse plus largement consacré à l'enseignement artistique supérieur, nous tenterons de mettre en lumière comment l'étudiant en art apprend peu à peu à devenir un artiste contemporain et donc un artiste théoricien. C'est que, comme l'indique Nathalie Heinich, concernant le paradigme de l'art contemporain, «aucune œuvre d'art contemporain, quel qu'en soit le genre (ready-made, art conceptuel, installation, performance et

1 France Nerlich, «Ateliers privés, enjeux et problématiques pour l'histoire de l'art du XIXe siècle », dans Alain Bonnet, France Nerlich (éds.), Apprendre à peindre, Tours, Presses universitaires François Rabelais, 2013, p. 18. 
même peinture) ne se présente dans le monde de l'art sans être accompagnée d'un discours. ${ }^{2}$ Certes, cette production du discours n'est pas forcément le fait de l'artiste lui-même et, même si c'est le plasticien qui produit ce discours, cela ne fait pas de lui un théoricien. Mais nous nous attacherons à démontrer comment cette production d'un discours, quel qu'il soit, pour accompagner la réalisation plastique, peut représenter pour les étudiants en art un décalage par rapport à leur représentation du travail artistique. En ce sens, cela crée bien un statut différent de celui de l'artiste plasticien et va dans le sens de l'idée de l'artiste théoricien. Nous y reviendrons. Nous serons également attentif aux différentes évolutions récentes dues au processus de Bologne et à la réforme LMD en ce qui concerne cette question de la théorie et, notamment, comment cela se traduit pour les étudiants en art. Ici, c'est surtout la question du travail de mémoire, désormais nécessaire pour obtenir un diplôme équivalent master, qui sera au centre de notre analyse.

Cette étude s'appuie sur un ensemble de données de type ethnographique, recueillies dans plusieurs écoles d'art françaises et auprès de différents acteurs de l'enseignement artistique supérieur. Ainsi, plusieurs périodes d'observations au sein de trois établissements différents nous ont permis de saisir au plus près la vie des ateliers et des salles de classe. Parallèlement, nous avons réalisé plus de quatre-vingt entretiens auprès d'étudiants, d'enseignants et de directeurs d'établissements. Ces entretiens semi-directifs et biographiques, avaient pour objectif de revenir sur le parcours des personnes interrogées et de mettre en lumière leurs représentations. Pour ce qui concerne le processus de Bologne, les observations comme les entretiens ayant été réalisés dans les premiers temps de l'application de la réforme, de nombreuses remarques et questions ont pu être soulevées par les enseignants ou les étudiants à ce sujet. Mais nous avons également eu l'opportunité de collecter les différents documents publiés par l'Agence d'évaluation de la recherche et de l'enseignement supérieur (AERES) dans le cadre de l'application de cette réforme, à la demande conjointe des ministères de la Culture et de la Communication et de l'Enseignement Supérieur et de la Recherche.

Dans un premier temps nous reviendrons sur la socialisation opérée dans les écoles supérieures d'art et plus particulièrement sur la place qu'occupe la production du discours théorique dans cette socialisation. Ensuite, nous présenterons en détail les différentes implications qu'a pu avoir le processus de Bologne pour les écoles d'art françaises.

La «fabrication » de l'artiste théoricien

2 Nathalie Heinich, Le paradigme de l'art contemporain, Paris, Gallimard, 2014, p. 175. 
Comme nous l'avons évoqué en introduction, l'enseignement délivré dans les écoles d'art est particulièrement orienté vers la théorie et correspond à une forme spécifique d'art relevant du paradigme de l'art contemporain. Pour le dire autrement, et pour reprendre les termes de Françoise Liot, les écoles d'art sont aujourd'hui quasi-exclusivement axées vers un «art discursif », forme d'art particulièrement adaptée au marché institutionnel et qui requiert de la part du créateur la production d'un discours afin de positionner et conceptualiser son travail plastique ${ }^{3}$. De fait, parce qu'il sera amené à produire de tels discours et à construire des dossiers, afin d'obtenir des financements, des résidences d'artistes et autres, le futur artiste se doit d'être familiarisé avec ce type d'activités en vue de sa future insertion professionnelle. Au-delà même de ces considérations pratiques, on peut avancer l'idée selon laquelle la création contemporaine accorde au discours et au concept un rôle particulièrement important, menant au fait que l'œuvre plastique ne peut plus se suffire à elle-même. Comme Pierre Bourdieu l'a écrit dans Les règles de l'art, « le travail artistique dans sa nouvelle définition rend les artistes plus que jamais tributaires de tout l'accompagnement de commentaires et de commentateurs qui contribuent directement à la production de l'œuvre par leur réflexion sur un art incorporant souvent lui-même une réflexion sur l'art, et sur un travail artistique comportant toujours un travail sur lui-même. » ${ }^{4}$ Ainsi, la «production de la croyance » est désormais au centre de la pratique artistique, production symbolique bien plus que matérielle qui vise à fonder la légitimité de l'œuvre et de l'artiste qui l'a produit. Or, cette façon de faire de l'art est en opposition avec les représentations profanes concernant l'art et les artistes. Là où le «grand public » valorise l'expression d'une sensibilité et le rapport authentique avec l'œuvre, la mise en avant du discours, qu'il soit du fait de l'artiste ou des critiques, apparaît comme une pratique superficielle, voire comme une escroquerie. Comme l'indique Nathalie Heinich à propos d'une commission municipale attribuant des subventions à des plasticiens, il y a renversement complet entre l'évaluation de ces professionnels de l'art (artistes, critiques, galeristes) et les jugements des profanes : «C'est, un peu, le monde à l'envers : ce qui apparaitrait comme inauthentique pour un public profane ("faire passer" une pratique artistique apparement indigente pour un discours prétendant à une ambition théorique) devient pour les spécialistes un critère d'authenticité si évident qu'il n'a pas besoin d'être justifié, tandis que son absence les plonge dans la même horreur, le même sentiment de fumisterie, qu'un spectateur moyen confronté à une ambition théorique sans savoirfaire proprement artistique. $»^{5}$ Or, pour ce qui concerne notre propre objet d'étude, ce renversement est particulièrement pertinent, dans la mesure où c'est celui que vivent les étudiants de manière très concrète au cours de leur passage dans les écoles d'art.

3 Françoise Liot, «L'école des beaux-arts face aux politiques de soutien à la création », Sociologie du travail, $\mathrm{n}^{\circ} 41-4$, 1999, p. 411-429.

4 Pierre Bourdieu, Les règles de l'art, Paris, Seuil, 1998, p. 24.

5 Nathalie Heinich, «Les frontières de l'art à l'épreuve de l'expertise. Politique de décision dans une commission municipale », Politix, n³8, 1997, p. 119. 
Certes, au moment de leur inscription aux beaux-arts, la plupart des étudiants ne sont pas complètement naïfs quant à la création artistique contemporaine. Beaucoup d'entre eux ont réalisé une ou deux années de classes préparatoires et nombreux sont ceux qui fréquentent les centres d'art contemporain. Pour autant, la représentation profane reste prégnante pour la plupart des étudiants, surtout en ce qui concerne la mise en avant du discours théorique. De fait, lorsqu'on interroge les étudiants sur les motivations qui les ont poussés à s'inscrire en école d'art, la grande majorité d'entre eux évoquent un rapport précoce avec la pratique artistique, notamment le dessin. Comme le rappelle Monique Segré dans sa propre étude des beaux-arts de Paris, beaucoup d'étudiants «se disent “nés avec le pinceau ou le crayon". [...] L'image mythique de l'artiste prédestiné, élu par les dieux, est fortement enracinée en eux. ${ }^{6}$ Par conséquent, leur rapport avec l'art découle avant tout de ce lien avec la pratique et c'est, pour la plupart d'entre eux, un talent particulier dans ce domaine (le fameux «coup de crayon ») qui les a poussé à s'orienter dans une carrière artistique. De même, le rapport authentique à la création plastique semble pour ces étudiants essentiel et ils conçoivent leur propre production comme l'expression immédiate de leur sensibilité. On comprend aisément comment de jeunes étudiants, âgés de moins de vingt ans, peuvent souscrire sincèrement à ce type de représentations romantique de l'artiste et insister sur le fait que leur œuvre est «le produit de leurs tripes ». Or, selon Nathalie Heinich, c'est justement sur cette question du lien entre intériorité de l'artiste et œuvre plastique que se fonde la rupture entre paradigme moderne et paradigme contemporain $^{7}$. Comme en attestent les ready made de Marcel Duchamp ou les monochromes d'Yves Klein, l'art contemporain a en effet largement remis en cause ce lien entre le créateur et sa création. Dès lors, au fur et à mesure qu'ils découvrent ces nouvelles façons de faire propres au paradigme contemporain, les étudiants en art peuvent dénoncer ce qui leur apparaît comme une dévalorisation de leur vocation originelle.

Ce renversement des valeurs et représentations que vivent les étudiants en art s'apparente à ce qu'Everett C. Hughes ${ }^{8}$ a identifié comme le «passage à travers le miroir » que connaissent les étudiants en médecine pendant leurs études. Selon le sociologue, au cours de la socialisation, les étudiants passent du monde profane au monde professionnel. Ils doivent dès lors abandonner les stéréotypes profanes concernant la profession et découvrent peu à peu une vision beaucoup plus désenchantée des activités et valeurs du groupe professionnel. Cette découverte peut être particulièrement troublante pour certains étudiants, fortement attachés à la représentation idéalisée

\footnotetext{
6 Monique Segré, L'art comme institution, l'école des beaux-arts (XIXe-XXe siècle), Paris, Éditions de l'ENS Cachan, 1993, p. 165.

7 Nathalie Heinich, op. cit., 2014, p. xx.

8 Everett C. Hughes, «The making of a physician », Human Organization, n 14,1955 , p. 21-25.
} 
de la profession, mais, comme le souligne Everett C. Hughes, elle peut également être excitante et enivrante pour d'autres étudiants qui adoptent la culture professionnelle et se l'approprient. Enfin, au cours de cette période, l'étudiant se doit d'alterner entre différents rôles. Ni tout à fait profane, ni tout à fait professionnel, l'individu lui-même et ses interlocuteurs ne savent pas à quelle identité il peut être renvoyé. Aux beaux-arts, ce «passage à travers le miroir » se joue notamment autour de cette question du discours accompagnant l'œuvre, ou pour le dire autrement, à propos de la « démarche » que l'étudiant doit développer. Ainsi, les étudiants apprennent peu à peu à considérer que les tâches qu'ils réalisent, parfois très éloignées de la peinture ou de la sculpture, ne sont pas forcément triviales mais peuvent avoir une légitimité artistique si tant est que l'étudiant produit la croyance dans cette démarche. Cette scène observée lors d'un workshop est significative sur ce point. Elle a eu lieu dans un terrain vague en Belgique, qui fit office de scène d'exposition pour les pièces réalisées in situ pendant le workshop. J'aidais alors un étudiant dans la réalisation de son travail, en cassant des briques et des tuiles à ses côtés. Notre but était ainsi de remplir un large carré au sol, avec ces gravas rouges, en guise d'installation. L'enseignant présent ce jour-là s'amusait de me voir travailler et plaisanta auprès de l'étudiant : «Quand il va rentrer Jérémie, il n'en reviendra pas de ce qu'il a fait, à casser des briques, pour une étude sur les beaux-arts ! ». Ce à quoi l'étudiant répondit : «À fond! Quand je parle de mes études à mon père, je lui dis que je casse des tuiles, ou que je porte des parpaings. Ben je fais les beaux-arts quoi ! Si la réponse humoristique de l'étudiant mérite d'être rapportée ici, c'est qu'elle témoigne parfaitement de la dualité dont parle Hughes à propos de la socialisation professionnelle. En effet, par le détour d'un autrui significatif (ici son père) représentant la vision profane, l'étudiant montre bien le décalage qui existe entre celle-ci et les tâches réellement effectuées. Par la même occasion, il signale à l'enseignant de quel côté de la barrière lui-même se situe, ayant bien compris que cette tâche-là ne constitue en rien l'essentiel du travail. À la fois profane (car comprenant l'étonnement possible de son père dans la mesure où il sous-entend) et professionnel (puisqu'il en plaisante), l'étudiant est inscrit dans une « série de choix de rôles » comme le souligne Everett C. Hughes. C'est que, comme le montre bien George Didi-Huberman ${ }^{9}$, l'art contemporain introduit un nouveau régime de croyance particulier. Il opère en effet le passage d'un « régime de tautologie » (l'objet n'est qu'un objet) à un « régime de croyance » (il y a en ceci autre chose que l'objet). C'est ce qu'a compris notre étudiant dans cette courte scène : en tant que professionnel, il sait bien que derrière cette activité triviale (casser des tuiles), il y a une opération symbolique et artistique, servant une démarche.

Ainsi, nous avons tenté de mettre en lumière de quelle façon s'opère la socialisation professionnelle aux beaux-arts et comment celle-ci se joue notamment autour de la «production de la croyance ».

9 George Didi-Huberman, Ce que nous voyons, ce qui nous regarde, Paris, Minuit, 1992. 
L'étudiant en art apprend ainsi au cours de ces études qu'il se doit de construire une « démarche » autour de laquelle s'articule ses productions plastiques. Pour ce faire, il doit appréhender une vision théorique de l'activité artistique, distincte de la seule maîtrise technique. C'est dans ce but que les écoles d'art fournissent un nombre considérable d'enseignements théoriques. Cela dit, la transmission de ces savoirs théoriques est toujours orientée vers la pratique et c'est justement ce qui est remis en cause par l'introduction récente du cadre LMD aux beaux-arts.

Le processus de Bologne et la question de la recherche

Le processus de Bologne a été initié en juin 1999 lorsque vingt-neuf ministres de l'enseignement supérieur signèrent la déclaration de Bologne, visant à l'uniformisation de l'enseignement supérieur en Europe, de manière à garantir une meilleure équivalence des formations et des diplômes sur le territoire de l'Union. Ensuite, cette déclaration a été introduite dans le droit français en avril 2002 et appliquée dans les universités sous le nom de réforme LMD (pour Licence Master Doctorat). Ce n'est qu'à partir de 2008 que cette réforme fit son entrée dans les écoles d'art et comme nous allons le constater, cela ne se fit pas sans heurts. Pour identifier quelles purent être les difficultés particulières qu'a pu causer la réforme LMD dans les écoles d'art, il peut être pertinent d'étudier tout d'abord les différentes recommandations produites par l'Agence de l'évaluation de la recherche et de la perspective (AERES) en janvier 2009 dans le cadre de son «évaluation prescriptive portant sur la possibilité d'attribution de grade de Master aux titulaires de DNSEP délivrés dans les écoles d'art ». Cette évaluation, commandée par la Délégation aux arts plastiques et la Direction générale de l'enseignement supérieur, résume parfaitement les différents points d'achoppement concernant l'application de la réforme dans les écoles supérieures d'art. Les débats ont d'ailleurs été vifs au sein même du comité d'experts et cela fut signalé dans le rapport lui-même, évoquant un «clivage [traduisant] en partie, mais en partie seulement, l'appartenance respective des experts à la communauté universitaire ou à celle des artistes, enseignants en école d'art. » Ainsi, deux points du rapport, concernant les critères pédagogiques pouvant faire obstacle à l'attribution du grade de Master, ne firent pas consensus au sein du comité et furent signalés comme tel. Le premier portait sur le mémoire de fin d'étude. De fait, un diplôme de Master requiert obligatoirement un mémoire réalisé par l'étudiant, comme c'est le cas à l'université. Pour citer le rapport de l'AERES, certains experts estimaient que le mémoire «ne saurait être un simple commentaire sur le travail plastique de l'étudiant [...] et répond, verbalement, à une démarche de recherche problématisée et à des exigences d'objectivation et de conceptualisation », $\mathrm{qu}^{\prime}$ «il doit être rédigé selon un plan raisonné et utiliser une bibliographie », qu'«il comporte au minimum 100000 signes [...] et respecte les normes requises pour les travaux de recherches universitaires. » Le second point de discorde 
concernait l'adossement de la recherche de la part des écoles d'art. Celui-ci est en effet jugé assez sévèrement par le comité d'experts, qui l'estime « insuffisant, voire très insuffisant. » Certes, et l'on voit bien tous les débats sous-jacents dans cette expression, le rapport souligne que l'on considère ici la recherche comme «comprise au sens universitaire du terme» mais il reste que la recommandation est ainsi établie. Le rapport indique en effet que «les écoles d'art ne pouvant à ce jour héberger de véritables équipes de recherche, les écoles sont invitées à établir des partenariats avec les équipes d'autres établissements d'enseignement supérieur et/ou à inciter leurs enseignants docteurs à intégrer ou s'associer à une équipe de recherche universitaire. » On le comprend bien, cette évaluation prescriptive de l'AERES tend à identifier la recherche avec le monde universitaire. Dès lors, elle semble imposer, ou du moins à encourager, le rapprochement des écoles d'art avec les formes et les critères de l'université, que cela concerne la forme du mémoire de fin d'études, le niveau des enseignants ou le lien avec les équipes de recherche. Or, c'est ce rapprochement qui fut largement remis en cause par une majorité des enseignants et étudiants en école d'art.

En janvier 2013, une tribune, publiée dans Libération et signée par l'ensemble des directeurs des écoles d'art françaises, indiquait : «les écoles d'art doivent garder leur singularité » Cette tribune, de même qu'un communiqué de l'Association nationale des directeurs d'écoles d'art (ANDEA) de juin 2013, furent publiées en réaction aux différents avis et projets de loi visant à établir une « cotutelle » du ministère de l'Enseignement supérieur sur les écoles d'art. Dès lors, les directeurs d'établissement signataires de cette tribune et du communiqué demandent l'annulation de cette cotutelle et le «rattachement exclusif » de leurs écoles au ministère de la Culture, se réclamant notamment d'une «exception culturelle ». Or, si ces revendications peuvent sembler accessoires visà-vis de ce que nous avons évoqué précédemment, il faut au contraire insister sur le fait qu'elles sont au centre du problème et témoignent bien des craintes que peuvent avoir les acteurs de l'enseignement culturel et artistique par rapport à la réforme. En effet, le principal objectif visé dans ce mouvement est le maintien de l'autonomie et des spécificités de ces écoles d'art, c'est-à-dire le maintien d'une démarcation claire avec l'enseignement supérieur généraliste et donc du monde universitaire. Même si les signataires estiment qu'il est nécessaire de maintenir des liens avec l'université sur certains points, laissant ainsi le dialogue ouvert pour ce qui concerne l'adossement à la recherche, ils visent tout de même clairement à ne pas se laisser absorber par le monde universitaire. Ces revendications ne sont pas nouvelles et se retrouvent même dans différents pays. Ainsi, aux débuts des années 1960 au Royaume-Uni, une réforme visant à remplacer le diplôme des écoles d'art (le NDD pour National Diploma of Design) par un diplôme plus généraliste calqué sur le modèle universitaire (Dip. AD pour Diploma in Art and Design) souleva le même genre de contestations de la part des enseignants en école d'art. Comme le montrent les auteurs de Art into 
$P o p^{10}$ à ce sujet, cette réforme fut fortement critiquée au nom de la spécificité de l'enseignement artistique qui ne pouvait se résoudre à se conformer à un système rigide laissant une trop grande place à la théorie aux dépens de l'expérimentation plastique. De la même manière, la refonte du système d'enseignement supérieur qui a pris place au Canada à la fin des années 1960 et qui a vu l'enseignement artistique entrer à l'université, s'est accompagnée de nombreuses négociations visant à faire reconnaître le statut particulier des artistes-enseignants et notamment autour de la définition de la recherche artistique ${ }^{11}$.

De fait, on retrouve le même genre de revendications chez les enseignants et les étudiants interrogés dans le cadre de cette étude. La question du mémoire a notamment cristallisé beaucoup de critiques, devenant le symbole de la réforme LMD. Les enseignants considèrent ainsi que les étudiants en art ne sont pas bien préparés à la réalisation d'un tel exercice, notamment s'il est obligatoire de suivre les canons universitaires recommandés par l'AERES. Certains estiment ainsi que ce mémoire empêchera certains étudiants, pourtant doués du point de vue de la création plastique, d'obtenir leur diplôme. En définitive, la réforme actuelle renvoie bien aux liens qu'entretiennent les écoles d'art avec le monde professionnel artistique. Le problème du mémoire se pose parce qu'il remet de fait en cause le critère artistique comme seul critère d'évaluation pertinent. Par conséquent, se pose aussi la question de la pertinence des évaluateurs. Comme l'ont bien montré les auteurs canadiens précédemment cités, le rapprochement entre le monde de l'art et celui de l'université amène à se poser la question des pairs: qui a la légitimité nécessaire pour juger le travail d'un artisteenseignant ? C'est le même problème qui surgit avec le mémoire de fin d'études ou avec l'autre volet de la réforme : l'adossement à la recherche. La nécessité pour les écoles de se lier à des laboratoires universitaires ou dans des institutions telles que les Pôles de recherche et d'enseignement supérieur (PRES) témoigne bien du fait que, pour ce qui concerne la recherche, les beaux-arts doivent se conformer à un modèle qui n'est pas forcément celui de la création artistique. Certains, dans les écoles d'art, peuvent être optimistes sur ce point, gageant que les échanges entre les deux univers amèneront à des formes innovantes. D'autres sont, on l'a vu, beaucoup plus critiques. Quoi qu'il en soit, ces débats autour de la réforme nous offre l'opportunité de mettre en lumière le statut spécifique de la théorie dans les écoles d'art. Ainsi, si l'apport des savoirs théoriques est considéré comme primordial par l'ensemble des enseignants (plasticiens comme théoriciens), il reste que celui-ci doit rester au service de la création plastique et ne peut donc pas emprunter les mêmes formes que les façons de faire universitaires. Il n'est pas de notre ressort ici de déterminer si la création artistique peut être considéré comme une forme de recherche théorique mais les différents

10 Simon Frith, Howard Horne, Art into Pop, London, Methuen, 1987, p. 40-43.

11 Mathurin Creutzer, Marcel Fournier, Yves Gingras, «Création artistique et champ universitaire : qui sont les pairs? », Sociologie et sociétés, n²1-2, 1989, p. 63-74. 
points soulevés par ce débat dans les écoles d'art constituent un apport pertinent à la question.

\section{Conclusion}

Si les écoles d'art forment des artistes, il est indéniable qu'elles apportent à ces derniers un large savoir théorique. L'art contemporain est, on l'a vu, indissociable de la question du discours, ou pour le dire plus précisément, de la démarche. La production de cette démarche, et la production de la croyance en cette démarche, est au cœur de l'activité artistique et ceci constitue une découverte pour les étudiants en art, qui peuvent s'en offusquer ou, au contraire, apprendre à jouer avec. C'est que les écoles d'art sont de fait des écoles particulières : le jeu avec les codes y est une pratique centrale. Il est de fait paradoxal de voir qu'un établissement scolaire encourage ses étudiants à contourner les règles. C'est d'ailleurs sans doute la raison pour laquelle le cadre rigide du mémoire universitaire est rejeté avec force dans les écoles. Mais c'est justement par ce jeu avec les règles et les conventions que les écoles d'art représentent une richesse pour l'élaboration de savoirs et d'outils théoriques différents. 